\title{
Importance of Benzothiazole Motif in Modern Drug Discovery: Introduction
}

\author{
Prakash Prajapat* \\ Department of Chemistry, Ganpat University, India
}

*Corresponding author: Prakash Prajapat, Department of Chemistry, Ganpat University, Mehesana-384012, Gujarat, India

Submission: 海 December 21, 2017; Published: 望 January 04, 2018

\begin{abstract}
Drug discovery \& their progress is major interest and present to the entire scientific community is a distilled version of newer developments in medicinal and pharmaceutical research area. Benzothiazoles are the most significant heterocyclic scaffold, which are widespread and integral feature of a broad range of natural occurring molecules and pharmaceutical agents. It shows a number of pharmacophoric activities, and its derivatives signify a high degree of structural diversity that has proven helpful for explore of newer bioactive agents.
\end{abstract}

Keywords: Drug design; Benzothiazole, Pharmaceuticals; Biological activity

\section{Introduction}

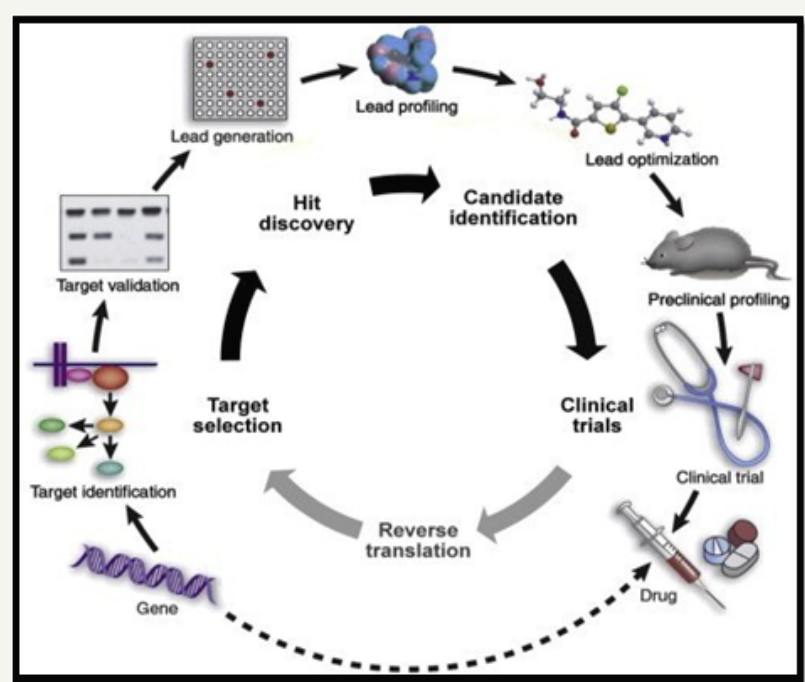

Figure 1: Ideal drug discovery and development process.

Heterocyclic molecules are pervasive in several areas of life sciences. These molecules perform numerous significant functions in nature, medicine, and technology. Heterocyclic hybrids have enormous potential as the most promising molecules as lead structures for the design of novel drug candidates in the field of modern drug discovery (Figure 1). The development of newer drug and pharmaceuticals is currently a critical and challenging issue to the academic researchers and pharmaceutical industry $[1,2]$. A wide range of synthetic and medicinal properties shown by heterocyclic hybrids inspired organic and medicinal chemists to pursue the synthesis of newer motifs and screen their pharmacophoric properties. Nitrogen-containing heterocycles specially azoles family are the subject of continuous interest in organic synthesis owing to the fact that they occur ubiquitously in pharmacologically active natural products, multipurpose oriented functional materials as well as highly potent pharmaceuticals and agrochemicals $[3,4]$. Benzothiazoles found to be most interesting biophore in research because it is used as a synthon for the synthesis of bioactive structures [5]. It is present in compounds involved in research aimed at evaluating new products that possess interesting biological activities viz. antitumor [6,7], antimicrobial [8-10], anti-inflammatory [11], anti-tubercular [12,13], antiHIV [14], anti-malarial [15], anti-convulsant [16] , anthelmintic $[17,18]$, anti-oxidant [19] and analgesic. The versatile synthetic applicability of bioactive heterocyclic scaffold help in the organic synthesis and pharmaceutical chemists to plan organize \& implement new strategies towards the drug discovery of newer benzothiazoles. In addition, the benzothiazole system is present in various marine or terrestrial natural compounds, which have useful biological activities. A variety of 2-aminobenzothiazoles have been described as central muscle relaxants and found to interfere with glutamate neurotransmission in biochemical, electrophysiological and behavioral experiments. They have also found application in industry as antioxidants, vulcanization accelerators [20]. Substituted benzothiazoles received much attention due to unique structure and its uses as imaging agents for $\beta$-amyloid plaques, photosensitizers, inhibitors of stearoyl-coenzyme A $\delta$ - 9 desaturase, LTD-4 receptor antagonist, orexin receptor antagonist and also a derivative of benzothiazole is the light-emitting component of luciferin, found in fireflies. Some important and clinically used 
drugs having benzothiazole ring in their structures are Riluzole, Thioflavin, Pittsburgh compound B, Ethoxzolamine, Pramipexole, Dimazole, Flutemetamol and Dithiazanine Iodide (Figure 2). Novel approach for benzothiazole motif as a biophore opens the door to the design, synthesis bio-evaluation of a variety of novel molecular hybrids. Hence, this information would give rise to design of better molecules with enhanced biological properties and higher specificity, and together with development of novel synthetic strategies.

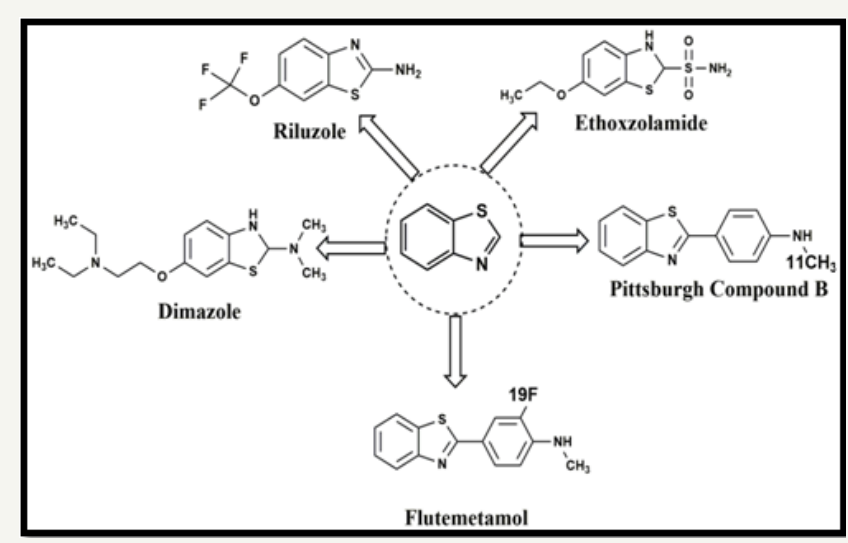

Figure 2: Several drugs containing Benzothiazole motif.

\section{Discussion \& Conclusion}

Drug discovery and development is and always has been the most exciting part of agrochemical and pharmaceutical industry. Organic synthesis has accredited a lot concern in recentyears, mostly those containing more than one heterocyclic ring in a molecule. Benzo-fused nitrogen, sulfur and oxygen-containing heterocyclic ring systems like benzothiazole, benzimidazole, benzoxazole are distinguished by a wide-ranging of high bioactivity, and therefore, they are interesting molecules for medicinal and pharmaceutical chemistry. These main pharmacophores are contained in a vast number of biologically active molecules that are used in agriculture and pharmaceuticals. Finally, this communication helps to find potential future directions on the drug design and development of new effective and specific analogues of azoles family specially benzothiazole for different biological targets or receptors.

\section{Acknowledgement}

I thankful to chemistry staff members of the Mehesana Urban Institute of Sciences, Ganpat University, Mehsana, Gujarat for their support.

\section{References}

1. Prajapat P, Vaghani H, Agarwal S, Talesara GL (2017) Synthetic and medicinal chemistry in drug discovery: needs for today. Ann Med Chem Res 3(1): 1021.

2. Prajapat $P$ (2017) Utility of drug discovery in medicinal and organic chemistry. Mod Chem Appl 5(4): e123.

3. Prajapat P, Talesara GL (2016) Synthesis and anti-inflammatory screening of some mono and bis-alkoxyphthalimide linked benzimidazole and their quinazoline and pyrimidine derivatives. J Heterocyclic Chem 53(5): $1603-1610$
4. Prajapat P, Kumawat M, Kherodiya B, Talesara GL (2016) An expedient synthesis and antimicrobial evaluation of ethoxyphthalimido derivatives of pyrimido [4,5-e]pyrimidine analogues from 1-(1H-benzimidazol-2yl)guanidine. J Indian Chem Soc 93: 539-544.

5. Agarwal S, Kalal P, Gandhi D, Prajapat P (2017) Thiazole containing Heterocycles with CNS activity. Curr Drug Discov Technol 14: 2017.

6. Kumbhare RM, Kosurkar UB, Ramaiah MJ, Dadmal TL, Pushpavalli SN, Bhadra MP (2012) Synthesis and biological evaluation of novel triazoles and isoxazoles linked 2-phenyl benzothiazole as potential anticancer agents. Bioorg Med Chem Lett 22(17): 5424-5427.

7. Hiyoshi H, Goto N, Tsuchiya M, Iida K, Nakajima Y, et al. (2014) 2-(4-Hydroxy-3-methoxyphenyl)-benzothiazole suppresses tumor progression and metastatic potential of breast cancer cells by inducing ubiquitin ligase CHIP. Sci Rep 4: 7095.

8. Prajapat P, Rathore KK, Gandhi D, Agarwal S, Hussain N, et al. (2016) A facile synthesis of biologically significant 2-(1,3-benzothiazol2-ylimino)-1,3-thiazolidin-4-one / / 3-(1,3-benzothiazol-2-yl)-2thioxoimidazolidin-4-on analogues from 1-(1,3-benzothiazol-2-yl) thiourea and their alphahydroxylamine derivatives. Iranian Journal of Organic Chemistry 8(2): 1795-1801.

9. Cano NH, Ballari MS, Lopez AG, Santiago AN (2015) New synthesis and biological evaluation of benzothiazole derivates as antifungal agents. J Agri Food Chem 63(14): 3681-3686.

10. Patel RV, Park SW (2015) Catalytic N-formylation for synthesis of 6-substituted-2-benzothiazolylimino-5-piperazinyl-4-thiazolidinone antimicrobial agents. Res Chem Intermed 41(8): 5599-5609.

11. Mahtab R, Srivastava A, Gupta N, Kushwaha SK, Tripathi A (2014) Synthesis of novel 2-benzylbenzo[d] thiazole-6-sulfonamide derivatives as potential anti inflammatory agent. J Chem Pharma Sci 7: 34-38.

12. Hazra K, Nargund LVG, Rashmi P, Chandra JNNS, Nandha B, Harish MS (2012) Synthesis and comparative study of anti-mycobacterium activity of a novel series of fluoronitrobenzothiazolopyrazoline regioisomers. Arch Pharm Chem Life Sci 345(2): 137-146.

13. Mir F, Shafi S, Zaman MS, Kalia NP, Rajput VS, et al. (2014) Sulfur rich 2-mercaptobenzothiazole and 1,2,3-triazole conjugates as novel antitubercular agents. Eur J Med Chem 76: 274-283.

14. Xu YS, Zeng CC, Jiao ZG, Hu LM, Zhong RG (2009) Design, synthesis and anti-HIV integrase evaluation of 4-oxo-4H-quinolizine-3-carboxylic acid Dderivatives. Molecules 14(2): 868-883.

15. Takasu K, Inoue H, Kim H, Suzuki M, Shishido T, et al. (2002) Rhodacyanine dyes as antimalarials. Preliminary evaluation of their activity and toxicity. J Med Chem 45(5): 995-998.

16. Siddiqui N, Rana A, Khan SA, Haque SE, Alam MS, et al. (2009) Synthesis of 8-substituted-4-(2/4-substituted phenyl)-2H-[1,3,5]triazino[2,1-b] [1,3] benzothiazole-2-thiones and their anticonvulsant, anti-nociceptive, and toxicity evaluation in mice. J Enz Inhib Med Chem 24(6): 1344-1350.

17. Munirajasekhar D, Himaja M, Malib SV (2014) A facile and efficient synthesis of 2-(5-(4-Substitutedphenyl)-4, 5-dihydro-3-phenylpyrazol1-yl)-6-substituted benzothiazoles and their biological studies. J Heterocyclic Chem 51(2): 459-465.

18. Sarkar S, Dwivedi J, Chauhan R (2013) Synthesis of 1-[2 (substituted phenyl)-4-oxothiazolidin-3-yl]-3-(6-fluro-7-chloro-1,3-benzothiazol-2yl)-ureas as anthelmintic agent. J pharm Res 7(5): 439-442.

19. Gouda MA, Eldien HF, Girges MM, Berghot MA (2013) Synthesis and antioxidant activity of novel series of naphthoquinone derivatives attached to benzothiophene moiety. Med Chem 3(2): 2228-2232.

20. Kumawat M, Kherodiya B, Prajapat P, Talesara GL (2015) Synthesis of alkoxyphthalimide derivatized oxoimidazolidinyl oxazolo/thiazolo dihydropyrimidine and oxoimidazolidinyl tetrahydropyrimidine via common Schiff base intermediate and evaluation of their antibacterial activity. Indian J Chem 54B(1): 117-127. 